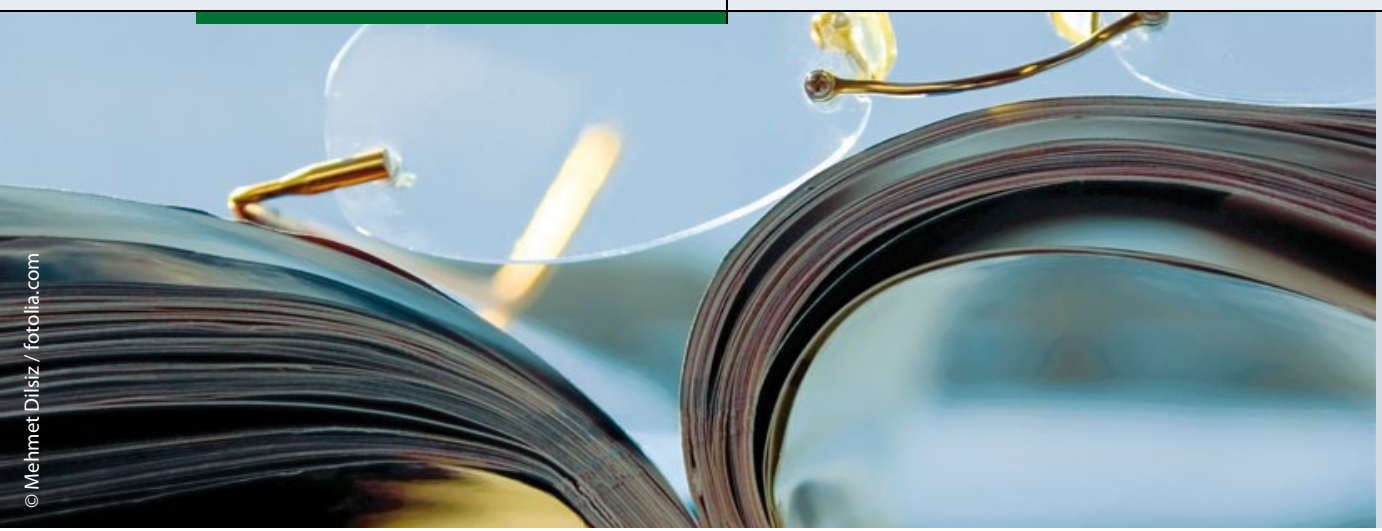

\section{Androgendeprivation: die Rolle der sexuellen Orientierung}

Die hetero- oder homosexuelle Orientierung von Männern hängt dem Konzept der "dualen Sexualität" zufolge mit unterschiedlichen Wirkungen von Östrogenen und Androgenen in den zerebralen Erregungszentren zusammen. Dies soll sich auch in unterschiedlichen Effekten einer Androgendeprivation äußern, wie sie in der Therapie des Prostatakarzinoms angewendet wird.

A $\mathrm{n}$ einer einschlägigen Studie eines rumänisch-amerikanischen Forscherteams hatten sich 29 Männer beteiligt, die wegen eines lokal fortgeschrittenen Prostatakarzinoms mit dem Antiandrogen Bicalutamid behandelt wurden. 17 der Männer waren hetero- und 12 homosexuell. Die Probanden sollten ihr Sexualleben anhand des „International Index of Erectile Function" (IIEF) bewerten. Verglichen wurden die Werte vor und fünf Wochen nach Beginn der Antiandrogentherapie.

Während sich beide Gruppen in den IIEF-Scores vor der Therapie nicht unterschieden, entwickelten sich unter der Therapie deutliche Differenzen zwischen hetero- und homosexuellen Männern. Die heterosexuellen Männer bemerkten hinsichtlich erektiler Funktion, Orgasmusfähigkeit, sexuellen Verlangens und allgemeiner Verkehrslage kaum Unterschiede zwischen Vorher und Nachher. Die homosexuellen Studienteilnehmer hingegen berichteten über teils deutliche Einbußen in nahezu allen Bereichen ihrer Sexualität:

-Der IIEF-Gesamtscore sank auf 28,7

Punkte (vorher: 52,9/maximal möglicher Wert: 75);

- die erektile Funktion wurde mit nur noch 12,1 bewertet $(24,5 / 30)$;
- die Orgasmusfunktion fiel auf 3,3 Punkte zurück $(7,2 / 10)$;

_das Verlangen sank auf 3,7 Punkte $(7,6 / 10)$;

_die Zufriedenheit mit dem Verkehr erhielt noch 6,2 Punkte $(8,0 / 10)$;

_die allgemeine sexuelle Zufriedenheit erreichte nur noch 3,4 Punkte $(7,1 / 10)$. Die Wissenschaftler schließen daraus, dass sich Androgene je nach sexueller Orientierung unterschiedlich auf die sexuelle Erregung auswirken. Stichwort ist hier die „duale Sexualität“. Dieser Hypothese zufolge existieren zwei neuroendokrine Achsen der Sexualität. Die eine soll bei heterosexuellen Frauen und homosexuellen Männern aktiv sein, wobei männliche Pheromone für die Libido und androgene Hormone für die Erregbarkeit zuständig wären. Die andere Achse würde weibliche Pheromone und Östrogene verbinden und die Sexualität von heterosexuellen Männern sowie lesbischen Frauen bestimmen. Generell wäre Homosexualität in diesem Modell des psychosexuellen Dualismus als Asynchronie zwischen den Genen, die das Geschlecht bestimmen, und jenen, die für die aktive sexuelle Achse zuständig sind, zu verstehen.

Mit einem praktischen Rat für die medizinische Forschung können die Studienautoren schließlich auch noch aufwarten.

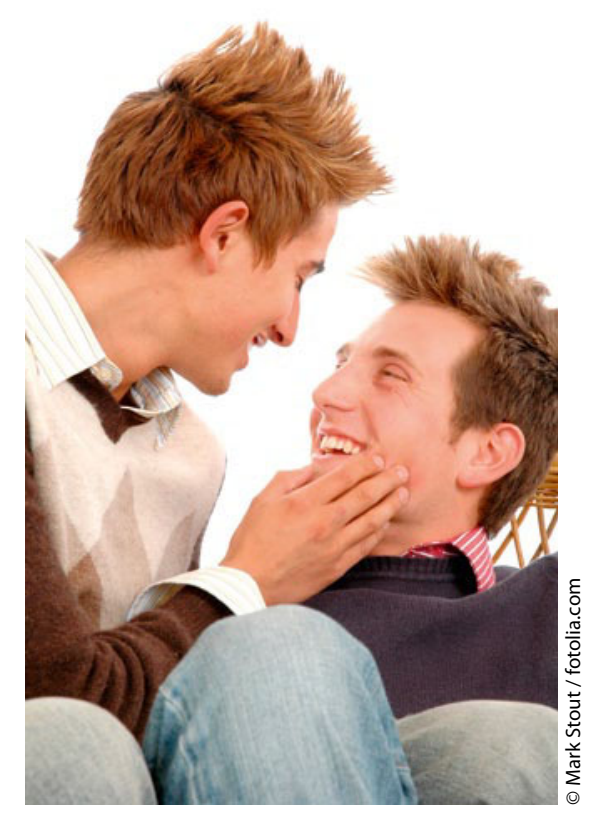

Homosexueller Männer sind laut der Theorie des psychosexuellen Dualismus stärker von Androgeneffekten abhängig als heterosexuelle. Androgensuppression setzt ihnen deshalb mehr zu.

Sie fordern, in Studien, die den Effekt bestimmter Substanzen auf das Sexualleben untersuchen, auch die sexuelle Orientierung der Probanden zu berücksichtigen.

Fazit: Androgene sind laut der vorliegenden Studie für die sexuelle Erregung homosexueller, nicht aber heterosexueller Männer zuständig, was Folgen für die Therapie des Prostatakarzinoms haben könnte. Das Sexualleben homosexueller Männer leidet deutlich mehr unter einer Behandlung mit Antiandrogenen. Dr. Robert Bublak

Motofei IG et al. Preliminary study with bicalutamide in heterosexual and homosexual patients with prostate cancer: a possible implication of androgens in male homosexual arousal. BJU Int 2011; 108: 110-5 